\title{
Comprehensive Analysis of LIN28A in Chinese Patients With Early Onset Parkinson's Disease
} Xiaojing Gu, Yanbing Hou, Yongping Chen, Ruwei Ou, Bei Cao, Qianqian Wei, Lingyu Zhang,
Wei Song, Bi Zhao, Ying Wu and Huifang Shang*

Laboratory of Neurodegenerative Disorders, Rare Disease Center, Department of Neurology, West China Hospital, Sichuan University, Chengdu, China

A loss-of-function variant in Lin-28 Homolog A gene (LIN28A p. R192G, rs558060339) has been identified in two East Asian ancestry patients with earlyonset PD (EOPD). Functional studies revealed that such a variant could lead to developmental defects and PD-related phenotype, and the phenotypes could be rescued after correction of the variant. The aim of the study was to screen the variants of LIN28A in Chinese patients with EOPD. A total of 682 EOPD patients were sequenced with whole exome sequencing and the coding and flanking region of LIN28A were analyzed. We identified a rare coding variant, $\mathrm{p}$. P182L, of LIN28A in a Chinese patient with EOPD. Moreover, we also found a $3^{\prime}$-UTR polymorphism (rs4659441) to be associated with an increased risk for PD. However, our rare variant burden analysis did not support a role for LIN28A as a major causal gene for PD.

Keywords: LIN28A, early onset Parkinson's disease, rare variant, screening, burden analysis

\section{INTRODUCTION}

Parkinson's disease (PD) is the second most common neurodegenerative disease with a complex spectrum of etiologies including aging, environmental factors, and genetic causes (Poewe et al., 2017). So far, more than 30 genes have been identified to be causative genes for PD, however, these could only explain a small proportion of patients (Shadrina et al., 1016).

With the development of genetic sequencing methods and bioinformatic analysis algorithms, novel $\mathrm{PD}$ causative genes have been extensively explored in recent years, and Lin-28 Homolog A gene (LIN28A) is one of them. LIN28A is a highly conserved RNA-binding protein, which is mainly expressed in the nervous system during early embryonic and fetal development and is involved in neuronal differentiation, tissue repair, and the maintenance of synaptic plasticity (Shinoda et al., 2013; Hu et al., 2020). Moreover, previous studies have also indicated that LIN28A might play a role in the pathogenesis of PD. For example, a previous study showed that LIN28A exhibits a strong therapeutic potential in the cell model and mouse model of PD (Rhee et al., 2016). A loss-of-function variant in the LIN28A (p.R192G, rs558060339) was identified in two East Asian ancestry patients with early-onset PD (EOPD) (Chang et al., 2019); developmental defects and PD-related phenotype due to the variant was rescued after correction of the variant (Chang et al., 2019). However, a subsequent study conducted in a large cohort of PD patients of European ancestry failed to find evidence supporting the causative role of LIN28A in PD (Diez-Fairen et al., 2021). There exists genetic 
heterogeneity among different regions. EOPD is more susceptible to genetic factors. Therefore, it is necessary to further investigate the role of LIN28A in EOPD.

\section{MATERIALS AND METHODS}

\section{Patients}

A total of 682 EOPD patients (age of onset $\leq 45$ years) admitted to the Department of Neurology, West China Hospital were recruited into the study. All the PD patients were diagnosed by experienced neurologists based on the established clinical diagnostic criteria for PD (Hughes et al., 1992; Postuma et al., 2015). Data of demographic, clinical characteristics, and rating scale assessments for patients were collected by face-to-face interview as previously described (Gu et al., 2020). Written informed consent was obtained from all participants. The study was approved by the ethics committee of West China Hospital, Sichuan University.

\section{Variant Selection}

Genomic DNA was collected from peripheral blood leukocytes and underwent whole exome sequencing (WES). Procedures of WES and variants annotation were performed as previously described ( $\mathrm{Gu}$ et al., 2020). All variants in the coding region and the flanking region of LIN28A captured by WES were included in the analysis. For the pathogenicity analysis, patients with mutations in known PD causative genes were excluded first.

\section{Single Nucleotide Polymorphism Association Analysis}

Controls were from the gnomAD East Asian population (https://gnomad.broadinstitute.org/). The genotype and allele frequencies were compared between the patients and controls using a chi-square test, and a $p$ value $<0.0056(0.05 /$ 9) was considered as statistically significant after Bonferroni correction.

\section{Rare Variants Burden Analysis}

Controls were from the gnomAD East Asian population (https://gnomad.broadinstitute.org/). All the rare coding variants annotated as "missense," "splice donor," "splice acceptor," "splice region," "stop-gained," or "in-frame deletion" were included. Rare variants were defined by minor allele frequency (MAF) lower than $0.1 \%$ in the public database as mentioned above. Combined Annotation Dependent Depletion (CADD) integrates predictions from numerous bioinformatic algorithms into a single "C-score" and ranks all possible nucleotide changes in the genome based on potential to disrupt gene/ protein function (Robak et al., 2017). In accordance with the previous study, we defined a stringent CADD C-score $\geq 12.37$ as likely damaging variants, representing the top $2 \%$ most damaging of all possible nucleotide changes in the genome-this subset is enriched for known pathogenic alleles (Uitterlinden et al., 2017). All the rare and rare deleterious variants annotated as "missense," "splice donor," "splice acceptor," "splice region," "stop-gained," or "in-frame deletion" were included. Five different algorithms method were used for burden analysis with AssotesteR Package in the rare variants level and rare deleterious variants level (Lee et al., 2014), including Comprehensive Approach to Analyzing Rare Genetic Variants (CARV), Sum of Squared Score (SSU), Sum Test (SUM), Cumulative Minor Allele Test (CMAT), and Bayesian Score Test (BST).

\section{RESULTS}

The coding region and the flanking region of LIN28A were captured by the WES. The likely pathogenic variant p. R192G was not detected in our cohort. In total, nine variants were detected in our cohort, including six intronic variants, a variant in the $3^{\prime}$-untranslated region $\left(3^{\prime}\right.$-UTR), a synonymous variant in exon 3 (c.270T > A, p. G90 =), and a missense variant in exon 4 (c.545C > T, p. P182L) (Table 1). The frequency of the missense variant is $0.0001(2 / 17,248)$ in the gnomAD East Asian controls (PM2). The variant was predicted to be tolerated among several in silico prediction tools including SIFT (tolerated), PolyPhen2_HDIV (benign), PolyPhen2_HVAR (benign), and LRT (Neutral). Therefore, it was considered to be a variant of uncertain significance (VUS) (Richards et al., 2015). The patient carrying the p. P182L developed tremor and rigidity at the age of 16. He first visited our clinic at the age of 23 years old, and was comprehensively assessed. His Unified Parkinson's Disease Rating Scale Part III score was 50, Horhn-Yahr stage was stage 3 , and cognition was normal.

In addition to the coding variants, there were seven noncoding variants identified in the flaking region. At the allelic level, rs4659441 in the $3^{\prime}$-UTR was found to be associated with an increased risk for EOPD (Table 1).

However, in the gene-based rare variant burden analysis, there was no significant enrichment of rare coding variants/rare damaging coding variants in Chinese patients with EOPD when compared with the gnomAD East Asian controls (Supplementary Table S1 and Supplementary Table S2).

\section{DISCUSSION}

In the current study, we identified a rare coding variant $\mathrm{p} . \mathrm{P} 182 \mathrm{~L}$ in the LIN28A in a Chinese patient with juvenile onset PD. Moreover, we found a polymorphism in the $3^{\prime}$-UTR to be associated with an increased risk for PD. However, rare coding variant burden analysis did not support LIN28A as a major causative gene for PD.

The rare coding variant p. P182L identified in our study, together with the previously described loss-of-function variant $\mathrm{p}$. R192G found in two East Asian ancestry patients with EOPD (Chang et al., 2019), and the rare coding variant p. T189I identified in a European PD patient (Diez-Fairen et al., 2021), were all located in the exon 4 of LIN28A, which is the C-terminal 
TABLE 1 | The description of variants in LIN28A identified in the EOPD patients.

\begin{tabular}{|c|c|c|c|c|c|c|c|c|c|}
\hline & gDNA & $\begin{array}{l}\text { Amino } \\
\text { acid } \\
\text { change }\end{array}$ & dbSNP147 & $\begin{array}{l}\text { Frequency } \\
\text { in patients }\end{array}$ & $\begin{array}{c}\text { Frequency } \\
\text { in gnomAD } \\
\text { east Asia }\end{array}$ & Class & Status & $\mathbf{p}$ & $\begin{array}{c}\text { Odds ratio } \\
\text { (95\% confidence } \\
\text { interval) }\end{array}$ \\
\hline $\begin{array}{l}1: \\
26,738,110\end{array}$ & $C>G$ & - & rs202211941 & $0.00586(8 / 1,364)$ & $0.00013(27 / 15378)$ & intronic & heterozygous & 0.006 & $3.354(1.521-7.398)$ \\
\hline $\begin{array}{l}1: \\
26,738,112\end{array}$ & $\mathrm{~T}>\mathrm{C}$ & - & - & $0.00073(1 / 1,364)$ & - & intronic & heterozygous & - & - \\
\hline $\begin{array}{l}1: \\
26,738,117\end{array}$ & $G>A$ & - & - & $0.00073(1 / 1,364)$ & - & intronic & heterozygous & - & - \\
\hline $\begin{array}{l}1: \\
26,738,122\end{array}$ & $\mathrm{C}>\mathrm{T}$ & & rs4623750 & $0.06871(94 / 1,364)$ & $0.08406(1,250 / 14870)$ & intronic & heterozygous & 0.052 & $0.806(0.649-1.002)$ \\
\hline $\begin{array}{l}1: \\
26,738,123\end{array}$ & delA & - & - & $0.00073(1 / 1,364)$ & - & intronic & heterozygous & - & - \\
\hline $\begin{array}{l}1: \\
26,751,835\end{array}$ & $\mathrm{~T}>\mathrm{A}$ & p.G90 = & - & $0.00073(1 / 1,364)$ & - & synonymous & heterozygous & - & - \\
\hline $\begin{array}{l}1: \\
26,751993\end{array}$ & $G>A$ & - & rs187064721 & $0.00147(2 / 1,364)$ & $0.00413(71 / 17184)$ & intronic & heterozygous & 0.052 & $0.177(0.025-1.274)$ \\
\hline $\begin{array}{l}1: \\
26,752,864\end{array}$ & $\mathrm{C}>\mathrm{T}$ & p.P182L & rs769630938 & $0.00073(1 / 1,364)$ & $0.00011(2 / 17248)$ & missense & heterozygous & 0.204 & $6.362(0.573-69.814)$ \\
\hline $\begin{array}{l}1: \\
26,752,992\end{array}$ & $\mathrm{~T}>\mathrm{C}$ & - & rs4659441 & $0.15322(209 / 1,364)$ & 0.11879 (1947/16390) & $3^{\prime}-$ UTR & heterozygous & $<0.001$ & $1.132(1.150-1.567)$ \\
\hline
\end{tabular}

Adjusted $\mathrm{p}=0.05 / 9=0.0056$.

domain, distal to an RNA-binding Zn-knuckle domain (residues 138-176) (Chang et al., 2019). Therefore, exon 4 might be a mutated hot spot for LIN28A, although more functional studies are needed. Our patient carrying p. P182L had a much earlier age of onset (at the age of 16) than that of the Korean patient carrying p. R192G (at the age of 23 and 60).

In addition to the rare coding variants identified in the current study, rs4659441 in the $3^{\prime}$-UTR was found to be associated with an increased risk for EOPD. Although untranslated, the $3^{\prime}$-UTR is important in the regulation of mRNA-based processes including mRNA localization, mRNA stability, and translation (Mayr, 2019). More specifically, $3^{\prime}$ UTR can provide binding sites to certain miRNAs and lead to mRNA degradation, therefore inhibiting gene expression (Mayr, 2019). Via bioinformatic tools (http:// www.mirbase.org/search-rnacentral.shtml) (Kozomara et al., 2019), we found that rs4659441 was a binding site for both hsamiR-4476 and hsa-miR-505-5p. Interestingly, hsa-miR-505$5 \mathrm{p}$, also known as hsa-miR-505*, has been found to be alterregulated in a wide spectrum of neurological disorders including multiple sclerosis, myasthenic gravis, Alzheimer's disease, Fredrich Ataxia, and Lacunar stroke (http://biobigdata.hrbmu.edu.cn/nsdna/map. jsp?organism = Homo\% 20sapiens). In relation to $\mathrm{PD}$, hsa-miR-505-5p has been found to be upregulated in the cell model of PD ( $\mathrm{Li}$ et al., 2013), and in the brain of progressive supranuclear palsy, a parkinsonism plus syndrome (Ubhi et al., 2014). Therefore, it can be speculated that the hsa-miR-505-5p could regulate the expression of LIN28A via binding to the polymorphism of rs4659441 in the $3^{\prime}$-UTR, and thus contribute to the pathogenesis of $\mathrm{PD}$. However, further functional studies are needed to elucidate the role of rs4659441.
Last but not least, burden analysis is a method that calculates the aggregated effect of a gene on some diseases. However, our findings using mathematical methods did not indicate the enrichment of rare variants in LIN28A in EOPD patients, which is consistent with the negative findings from the European population (Diez-Fairen et al., 2021).

\section{CONCLUSION}

In conclusion, our findings of a rare coding variant $\mathrm{p}$. $\mathrm{P} 182 \mathrm{~L}$ expanded the mutation spectrum of LIN28A in EOPD. Moreover, we also found a risk allele for EOPD in the $3^{\prime}$-UTR. However, our rare variant burden analysis together with the findings from the European population did not support the hypothesis that LIN28A plays a major causative role in PD. More studies in different genetic backgrounds are needed.

\section{DATA AVAILABILITY STATEMENT}

The original contributions presented in the study are included in the article/Supplementary Material, further inquiries can be directed to the corresponding author.

\section{ETHICS STATEMENT}

The studies involving human participants were reviewed and approved by the ethics committee of West China Hospital, Sichuan University. The patients/participants provided their written informed consent to participate in this study. 


\section{AUTHOR CONTRIBUTIONS}

1. Research project: A: Conception and design; B: Acquisition of data; C: Analysis and interpretation of data; 2. Statistical Analysis: A. Design, B. Execution, C. Review and Critique; 3. Manuscript Preparation: A. Writing of the first draft, $B$. Review and Critique. $\mathrm{XG}$ : $1 \mathrm{~A}, 1 \mathrm{~B}, 1 \mathrm{C}, 2 \mathrm{~B}$, and $3 \mathrm{~A}$; $\mathrm{YH}$ : 1B, 1C, 2B, 2C, and 3B; YC: $2 \mathrm{C}$ and $3 \mathrm{~B}$; RO: $1 \mathrm{~B}$ and $2 \mathrm{~B}$; $\mathrm{BC}$ : $1 \mathrm{~B}$ and $2 \mathrm{~B}$; $\mathrm{QW}$ : $1 \mathrm{~B}$ and $2 \mathrm{~B}$; $\mathrm{LZ}$ : $1 \mathrm{~B}$ and $2 \mathrm{~B}$; WS: $1 \mathrm{~B}$ and $2 \mathrm{~B}$; $\mathrm{BZ}$ : $1 \mathrm{~B}$ and $2 \mathrm{~B}$; $\mathrm{YW}$ : $1 \mathrm{~B}$ and $2 \mathrm{~B}$; HS: $1 \mathrm{~A}$, $1 \mathrm{~B}, 2 \mathrm{~A}, 2 \mathrm{C}$, and $3 \mathrm{~B}$.

\section{FUNDING}

This work was supported by the National Key Research and Development Program of China (Grant No.2016YFC0901504),

\section{REFERENCES}

Chang, M. Y., Oh, B., Choi, J. E., Sulistio, Y. A., Woo, H. J., Jo, A., et al. (2019). LIN 28A Loss of Function Is Associated With Parkinson's Disease Pathogenesis. EMBO J. 38, 1-17. doi:10.15252/embj.2018101196

Diez-Fairen, M., Makarious, M. B., Bandres-Ciga, S., and Blauwendraat, C. (2021). Assessment of LIN28A Variants in Parkinson's Disease in Large European Cohorts. Neurobiol. Aging. 100, e1-118. doi:10.1016/ j.neurobiolaging.2020.12.002

Gu, X., Li, C., Chen, Y., Ou, R., Cao, B., Wei, Q., et al. (2020). Mutation Screening and Burden Analysis of VPS13C in Chinese Patients With Early-Onset Parkinson's Disease. Neurobiol. Aging. 94, e1-311. e4. doi:10.1016/ j.neurobiolaging.2020.05.005

$\mathrm{Hu}, \mathrm{Z}$., Ma, J., and Gu, Y. (2020). Lin28a Is Essential for Synaptic Plasticity in Dentate Granule Cells and Spatial Memory. Neurosci. Bull. 37, 261-266. doi:10.1007/s12264-020-00591-7

Hughes, A. J., Daniel, S. E., Kilford, L., and Lees, A. J. (1992). Accuracy of Clinical Diagnosis of Idiopathic Parkinson's Disease: a Clinico-Pathological Study of 100 Cases. J. Neurol. Neurosurg. Psychiatry. 55, 181-184. doi:10.1136/ jnnp.55.3.181

Kozomara, A., Birgaoanu, M., and Griffiths-Jones, S. (2019). MiRBase: From MicroRNA Sequences to Function. Nucleic Acids Res. 47, D155-D162. doi:10.1093/nar/gky1141

Lee, S., Abecasis, G. R., Boehnke, M., and Lin, X. (2014). Rare-Variant Association Analysis: Study Designs and Statistical Tests. Am. J. Hum. Genet. 95, 5-23. doi:10.1016/j.ajhg.2014.06.009

Li, L., Chen, H., Chen, F., Li, F., Wang, M., Wang, L., et al. (2013). Effects of Glial Cell Line-Derived Neurotrophic Factor on microRNA Expression in a 6Hydroxydopamine-Injured Dopaminergic Cell Line. J. Neural Transm. 120, 1511-1523. doi:10.1007/s00702-013-1031-z

Mayr, C. (2019). What Are $3^{\prime}$ UTRs Doing? Cold Spring Harb Perspect. Biol. 11, a034728-16. doi:10.1101/cshperspect.a034728

Poewe, W., Seppi, K., Tanner, C. M., Halliday, G. M., Brundin, P., Volkmann, J., et al. (2017). Parkinson Disease. Nat. Rev. Dis. Primers. 3, 17013. doi:10.1038/ nrdp. 2017.13

Postuma, R. B., Berg, D., Stern, M., Poewe, W., Olanow, C. W., Oertel, W., et al. (2015). MDS Clinical Diagnostic Criteria for Parkinson's Disease. Mov Disord. 30, 1591-1601. doi:10.1002/mds.26424

Rhee, Y.-H., Kim, T.-H., Jo, A.-Y., Chang, M.-Y., Park, C.-H., Kim, S.-M., et al. (2016). LIN28A Enhances the Therapeutic Potential of Cultured Neural Stem Cells in a Parkinson's Disease Model. Brain. 139, 2722-2739. doi:10.1093/brain/aww203 the 1.3.5 project for disciplines of excellence, West China Hospital, Sichuan University (ZYJC18038 and ZY2016203) and the Science Foundation of Chengdu Science and Technology Bureau (2019-YF05-00307-SN).

\section{ACKNOWLEDGMENTS}

The authors thank all the patients for their participation.

\section{SUPPLEMENTARY MATERIAL}

The Supplementary Material for this article can be found online at: https://www.frontiersin.org/articles/10.3389/fgene.2021.740096/ full\#supplementary-material

Richards, S., Aziz, N., Aziz, N., Bale, S., Bick, D., Das, S., et al. (2015). Standards and Guidelines for the Interpretation of Sequence Variants: a Joint Consensus Recommendation of the American College of Medical Genetics and Genomics and the Association for Molecular Pathology. Genet. Med. 17, 405-423. doi:10.1038/gim.2015.30

Robak, L. A., Jansen, I. E., van Rooij, J., Uitterlinden, A. G., Kraaij, R., Jankovic, J., et al. (2017). Excessive Burden of Lysosomal Storage Disorder Gene Variants in Parkinson's Disease. Brain. 140, 3191-3203. doi:10.1093/brain/awx285

Shadrina, M. I., Slominsky, P. A., Limborska, S. A., T.-I. R. of C., K. W. B., and Jeon, M. B. "Chapter 6 - Molecular Mechanisms of Pathogenesis of Parkinson's Disease," in International Review of Cell and Molecular Biology (Academic Press), 229-266. doi:10.1016/S1937-6448(10)81006-8

Shinoda, G., Shyh-Chang, N., Soysa, T. Y. d., Zhu, H., Seligson, M. T., Shah, S. P., et al. (2013). Fetal Deficiency of Lin28 Programs Life-Long Aberrations in Growth and Glucose Metabolism. Stem Cells. 31, 1563-1573. doi:10.1002/ stem. 1423

Ubhi, K., Rockenstein, E., Kragh, C., Inglis, C., Spencer, B., Michael, S., et al. (2014). Widespread MicroRNA Dysregulation in Multiple System Atrophy - DiseaseRelated Alteration in miR-96. Eur. J. Neurosci. 39, 1026-1041. doi:10.1111/ ejn. 12444

Uitterlinden, G., Robak, L. A., Jansen, I. E., Rooij, J. V., and Kraaij, R. (2017). Excessive Burden of Lysosomal Storage Disorder Gene Variants in Parkinson 'S Disease. Brain. 140, 3191-3203. doi:10.1093/brain/awx285

Conflict of Interest: The authors declare that the research was conducted in the absence of any commercial or financial relationships that could be construed as a potential conflict of interest.

Publisher's Note: All claims expressed in this article are solely those of the authors and do not necessarily represent those of their affiliated organizations, or those of the publisher, the editors and the reviewers. Any product that may be evaluated in this article, or claim that may be made by its manufacturer, is not guaranteed or endorsed by the publisher.

Copyright (c) 2021 Gu, Hou, Chen, Ou, Cao, Wei, Zhang, Song, Zhao, Wu and Shang. This is an open-access article distributed under the terms of the Creative Commons Attribution License (CC BY). The use, distribution or reproduction in other forums is permitted, provided the original author(s) and the copyright owner(s) are credited and that the original publication in this journal is cited, in accordance with accepted academic practice. No use, distribution or reproduction is permitted which does not comply with these terms. 